\title{
Clustered Ring Enhancement
}

National Cancer Institute

\section{Source}

National Cancer Institute. Clustered Ring Enhancement. NCI Thesaurus. Code C114965.

Thin rings of enhancement visible in MRI that are clustered together around breast ducts

in an area that is neither a mass nor a foci. 